\title{
En torno al sentido de gobernabilidad y gobernanza: delimitación y alcances"
}

\author{
Around the Sense of Governability and Governance: \\ Definition and Scope
}

JORGE BROWER BELTRAMIN**

\begin{abstract}
Resumen: durante las últimas décadas los conceptos de gobernabilidad y gobernanza han cobrado gran relevancia en el ámbito de las teorías políticas y las diversas reflexiones y aportes en relación al fortalecimiento de las democracias en todo el mundo. Este artículo establece un recorrido de lectura sobre la geografía conceptual de los términos gobernabilidad y gobernanza, exponiendo en ese recorrido vinculaciones significativas con otros campos de sentido y nociones al interior de ellos, que posibilitan una mejor comprensión de estos términos. Finalmente proponemos una relectura desde la complejidad para enriquecer el sentido de los conceptos gobernabilidad y gobernanza. Este aporte, proveniente de una dimensión gnoseológica epistémica contri-
\end{abstract}

\begin{abstract}
During the last decades, the concepts of governability and governance have become important in the field of political theory and the various reflections and contributions relating to the strengthening of democracies worldwide. This article establishes a course of reading on the conceptual geography of the terms governability and governance, in the journey exposing, significant linkages with other fields of meaning and concepts within them, which enables a better understanding of these terms. Finally, we propose a rereading from complexity to enrich the meaning of governability and governance concepts. This contribution, from a epistemic dimension contributes not only to increase the density of semantic concepts but also provides
\end{abstract}

Fecha de recepción: 07/07/2014. Fecha de aceptación: 05/08/2014.

* Este texto ha sido de gran relevancia para el desarrollo del referente teórico del proyecto de investigación: "Gobernanza para la Educación Superior en la administración Bachelet (2014-2018): exploración crítica discursiva de la reforma educacional” para el período académico 2015-16. Universidad de Santiago de Chile, Usach. Agradecimientos Proyecto DICYT,Código 031576BB, Vicerrectoría de Investigación, Desarrollo e Innovación.

** Universidad de Santiago de Chile. Académico Jornada Completa. Coordinador del área de investigación del Departamento de Publicidad e Imagen (DPI).jorge.brower@usach.cl. Líneas de investigación: Semiótica de La cultura, Análisis Crítico del discurso, Epistemología de las Ciencias Sociales. Publicaciones recientes: Brower, J. "Jaques Derrida, Universidad sin condición, Trotta, Madrid, 2010. (Trad. Cristina de Peretti y Paco Vidarte) 77 pp. Revista Diánoia (Scielo-México). Facultad de Filosofía y Letras. Universidad Nacional Autónoma de México. Vol. LVIII, N71, noviembre de 2013. Brower, J. "El encuentro de las culturas desde la perspectiva de Burkel The meeting of cultures from the perspective of Burke. Peter Burke, Hibridismo cultural. Ediciones Akal, Madrid, 2010 (Trad. Sandra Chaparro Martínez) 158 p.” Revista RUNA, archivo para las ciencias del hombre (Scielo-Argentina). Universidad de Buenos Aires. Facultad de Filosofía y Letras. Instituto de Ciencias Antropológicas. Vol. 33, N², 2012. 
buye no sólo a aumentar la densidad semántica de los conceptos sino que aporta una forma de conocimiento cuyo efecto va más allá del plano teórico, viéndose expresado en las políticas públicas y su implementación en sociedades concretas. Palabras clave: gobernabilidad, gobernanza, democracia, complejidad. a form of knowledge whose effect goes beyond the theoretical level, seeing expressed in public policies and their implementation in specific societies.

Keywords: governability, governance, democracy, complexity.

\section{Algunas señalizaciones respecto al recorrido conceptual}

En las últimas décadas han cobrado gran relevancia los conceptos de gobernabilidad y gobernanza, entendidos de modo general como aquella capacidad que tienen las sociedades para proveer un sistema de gobierno que permita el desarrollo de los sistemas sociales y obviamente, de los individuos que forman parte de ellos.

Desde nuestra perspectiva es muy relevante el sentido de cada uno de estos términos, así como los campos semánticos que se generan desde ellos o que sirven de plataforma para su expresión. En otras palabras, la puesta en discurso de gobernabilidad y gobernanza es la expresión visible de un conjunto de procesos sociopolíticos, de tensiones entre los diversos actores que asumen la tarea de gobernar (en niveles locales, nacionales, regionales y otros) y en definitiva, es la manifestación reconocible de estructuras ideológicas desde las cuales se plasma una visión de sociedad.

En este trabajo, haremos un recorrido sintético por las avenidas de sentido que se generan desde los conceptos de gobernabilidad y gobernanza, exponiendo sus significados centrales o fundamentales y las derivaciones que se proyectan desde ellos. Del mismo modo, exploraremos otros campos semánticos vinculados a la perspectiva epistemológica de la complejidad, que a nuestro juicio podrían conectarse a estas unidades expresivas aportando contenidos y perspectivas que explican fenómenos como los sociales y que por tanto enriquecerían la densidad de ambos conceptos.

\section{Gobernabilidad: centro y fronteras conceptuales}

Los estudios y análisis en el campo de las ciencias políticas y de otras disciplinas afines concuerdan en que el informe presentado por Crozier, Huntington y Watanuki (1975) a la Comisión Trilateral sobre la crisis de la democracia, representa un punto de inflexión importante en el que se instala en la agenda política internacional el concepto de gobernabilidad (gobernability). Factores tales como las crisis financieras, las demandas sociales cada vez más diversas y fragmentadas, unidas a acciones colectivas que han ido adquiriendo mayor visibilidad social, han exigido un replanteamiento de esta noción (gobernabilidad), que en definitiva permita hacerse cargo de los desafíos que la sociedad civil en su conjunto plantean a quienes gobiernan o a la clase gobernante. En este contexto, la gobernación (governing) o el gobierno, en cualquiera de sus dimensiones (local, nacional, etc.) debe ser capaz de hacer efectiva una gobernabilidad que avance y consolide los proyectos democráticos en el área económica y social. Dicho lo anterior, podríamos afirmar que el horizonte semántico más amplio y general respecto a la gobernabilidad tiene que ver, como señala Prats (2001), con 
la existencia de estabilidad política que garantice el desarrollo humano. Los procedimientos que aseguran estos principios de estabilidad y desarrollo deberán estar asociados a criterios de flexibilidad permanentes que sean capaces de recoger las demandas y conflictos de dinámicas sociales complejas.

En esta línea de exploración de los elementos de sentido nuclear que dan cuenta de la gobernabilidad, el concepto aparece también en términos de principio, como una condición necesaria para el desarrollo de las sociedades que debe ir acompañada de un sustento o sustrato democrático (Prats, 2001). Siguiendo con la delimitación del campo semántico de la gobernabilidad y los elementos de sentido a partir de los cuales este se articula, podemos enfatizar una propiedad que ya enunciábamos antes. La gobernabilidad significa inicial y sustantivamente la capacidad de los sistemas políticos para responder a las demandas sociales cada vez más diversas y complejas (Jiménez et al, 2007). A partir de esta delimitación conceptual básica e inicial, el concepto de gobernabilidad se vincula estrechamente al de democracia. De este modo, podemos observar que lo que se ha tratado de definir desde las teorías sociopolíticas, es el término que integra gobernabilidad y democracia. En efecto, estos dos conceptos entran en contacto ya en el informe de Crozier, Huntington y Watanuki (1975), cuya finalidad se orienta a las capacidades de gobernabilidad que exhiben las democracias de los países desarrollados. Como señala Jiménez (et al 2007), la gobernabilidad (al igual como sucede con la gobernanza), no es más que la expresión que refiere directamente a la forma de funcionamiento más o menos acertada de las democracias occidentales exigidas y tensionadas por una realidad compleja, convulsionada y mundializada.

No es el interés de este trabajo entrar exhaustivamente al campo de sentido que implica la noción de democracia, sin embargo podemos señalar que la teorización sobre la gobernabilidad la implica de manera insoslayable, entendiendo que este sistema político es el que debe proveer el conjunto de reglas mediante el cual se resuelvan los conflictos sociales y se establezcan los principios rectores para el desarrollo del ser humano. En este contexto, siguiendo la propuesta de poliarquía de Dahl (1998), la democracia debe asegurar los estándares de participación real, igualdad de voto, entendimiento informado, control final sobre la agenda e inclusión de los adultos. Estos elementos, fundamentales para la articulación de un concepto empírico de democracia, resultan ser el campo semántico-ideológico de base para que la gobernabilidad pueda funcionar y ser validada al interior de una sociedad. Esta vinculación entre gobernabilidad y democracia, en definitiva entre dos campos semánticos diferentes que aparecen como complementarios, es muy significativa en la reflexión y producción teórica sobre estos conceptos al aparecer ligados de manera casi natural. Los procedimientos que dan cuenta de una poliarquía (como apuntábamos con Dahl) se presentan como condición fundamental para la existencia de una gobernabilidad democrática. Dicho estado político de base permitiría que todos los actores estratégicos participen en la toma de decisiones y por otro lado exigiría a quienes ostentan poderes institucionalizados (económicos, políticos, religiosos, etc.), que acaten las resoluciones emanadas de los aparatos legislativos y ejecutivos dentro de un escenario que resguarde las libertades políticas y los derechos fundamentales de las personas.

Así como se observa esta fuerte vinculación entre gobernabilidad y democracia en la producción de las teorías politológicas, existe un segundo concepto y su correspondiente campo de sentido al que aparece ligada la gobernabilidad. Se trata de la economía de 
mercado entendida dentro del modelo neoliberal de desarrollo. Esta vinculación también es muy significativa, ya que desde el informe de Crozier (et al 1975), no sólo preocupa la crisis de la gobernabilidad democrática sino que también la preservación de la economía de mercado. Para estos autores, entre los que destaca Huntington (1972, 1991), el aparato del estado debe tener un conjunto de funciones mínimas, de tal forma que no entorpezca la producción y re-distribución de la riqueza. La gobernabilidad se carga, a través de esta vinculación conceptual, de un sentido orientado a la reducción del estado en términos de su intervención/regulación de los diferentes actores e instituciones sobre las que se articulan las sociedades.

Esta segunda vinculación (gobernabilidad-modelo neoliberal) ha tenido una metalectura crítica interesante desde América Latina. Autores como Kauffman (2002) han señalado que la corrupción en nuestra región ha dado paso a una mala gobernabilidad neoliberal. De allí su relectura de un buen gobierno y por consiguiente de una optimización de la gobernabilidad, tiene que ver con la práctica de la autoridad considerando las tradiciones e instituciones que se orientan al bien común. Todo esto en un marco de respeto a los ciudadanos en relación a las interacciones económicas y sociales que los involucren. En esta misma línea de relectura crítica/conflictivista, Rottman (2002) aporta una perspectiva que comprende la gobernabilidad dentro de modelos democráticos liberales sustentados a su vez en un modelo neoliberal de desarrollo.Esto se traduce, como hemos adelantado antes, en una concepción neutral del Estado dentro de la cual éste no debe intervenir en las dinámicas propias del mercado. Así comprendida la gobernabilidad (en el contexto latinoamericano), esta se ha traducido finalmente en una serie de operaciones normativas que sólo potencias la lógica desde la cual se potencia la sociedad del consumo. El propio Rottman (2002), concluye diciendo que en nombre de esta concepción de gobernabilidad se han establecido nuevas redes de explotación y nuevas formas de colonialismo en América Latina.

Como se puede observar, el concepto de gobernabilidad ha entrado en una dinámica de sentido con rasgos polisémicos importantes. Es claro que las asociaciones ideológicas desarrolladas respecto a esta noción le han ido dando un espesor o densidad semántica que la complejiza. Como advierte Prats (2001), el término se ha utilizado en variados campos y ámbitos, no teniendo en ellos una correspondencia que la defina en forma clara. Por otro lado, Prats (2001) agrega que la falta de una delimitación conceptual entre gobernabilidad y gobernanza (governance) hace necesario avanzar en la clarificación conceptual respecto de los campos de sentido de cada uno de ellos.

Desde nuestra perspectiva, sabemos que no existen conceptos estáticos respecto a su sentido y por tanto a su proyección connotativa. Al contrario, nos interesa este recorrido de lectura en tanto establece vinculaciones semánticas significativas y simultáneamente tensiona el término gobernabilidad,desplegando nexos de sentido que dan cuenta de toda una geografía discursivo/conceptual a través de la cual se intenta explicitar cómo es y cómo debe ser la acción de gobernar o la gobernación (governing) de sociedades complejas que, a través de sus demandas se encuentran más allá de cualquier aparato ideológico y sus correspondientes visiones de mundo sesgadas y clausurantes. La sociedad civil se desplaza hacia modalidades de desarrollo expresadas en demandas que constituyen una exigencia y al mismo tiempo un reto para quienes gobiernan, estableciendo condiciones de gobernabilidad que sean capaces de hacerse cargo de dichas demandas. 


\section{Gobernanza: despliegue de un campo discursivo más allá de la gobernabilidad}

La teoría sociopolítica ha evolucionado en el último tiempo desde el tema/concepto gobernabilidad, entendida de manera amplia como aquella capacidad de los sistemas políticos para hacerse cargo de las múltiples demandas sociales, hacia una teorización y por tanto una producción conceptual sobre la noción de gobernanza, referida específicamente a los procedimientos que posibilitan la interacción horizontal entre el ámbito público, el privado y los actores sociales, en el contexto más amplio de una institucionalidad que permita avanzar sobre grandes acuerdos sociales. Entendida en el campo específico de la gestión, el concepto de gobernanza (governance) es validado a partir de los informes del Banco Mundial (1989), sobre la crisis de desarrollo sufrida por la región del África Subsahariana. En tal contexto se advierte sobre la necesidad de una buena gestión pública a propósito de los altos niveles de corrupción y los procesos de privatización de las entidades públicas. En dicho informe, estos elementos son conectados con la adopción del modelo de economía de mercado sustentado en una ideología claramente neoliberal. Al igual que en el informe de Crozier (et al, 1975) aludido a propósito del concepto de gobernabilidad, el trabajo evacuado por el Banco Mundial (1989), se preocupa por el buen gobierno (good governance), es decir, por la gobernanza en el ámbito de las democracias liberales.

Una buena delimitación conceptual de gobernanza, es aportada por Mayntz (2000), quien pone énfasis en que el nuevo estilo de gobierno debe permitir grados crecientes de cooperación e interacción entre los diferentes actores de una sociedad, sean estos estatales, privados o de otro tipo de organizaciones u origen. Esta cooperación e interacción garantizarían la articulación de un nuevo orden social. De este modo, la gobernabilidad que ya hemos definido antes como aquella capacidad general de gobernar, sería dependiente de la variable más específica representada por el concepto de gobernanza tal como apuntan (Prats 2003) y Aguilar (2007) entre otros. Esto, ya que este último produce las reglas del juego para una institucionalidad en la que se hagan posibles el conjunto de interacciones e intercambios que finalmente dan cuenta de una mejor capacidad de gobierno.

Ahora bien, en términos de un despliegue de campos de sentido desde el concepto central de gobernanza, éste se encuentra vinculado a tres nociones que quisiéramos destacar. La primera de ellas tiene que ver con la red de políticas, elemento/característica que para autores como Gaudin (2002) y Zurbriggen (2003), constituye una cuestión fundamental para entender el concepto contemporáneo de gobernanza. Esta red de políticas tiene que ver básicamente con que las políticas públicas se deciden y ejecutan a partir de redes que se generan, a través de coordinaciones sociales en diversas dimensiones de la vida social, tales como el autogobierno o la producción de políticas contractuales, acciones propias de una coordinación horizontal y no de una práctica vertical de la autoridad. En esta primera vinculación, importa el concepto de red ya que caracteriza nuevas formas de gobierno más allá de los límites que propone/ impone el Estado y las regulaciones del mercado.

La segunda noción vinculada al concepto de gobernanza que queremos mencionar es la de políticas públicas. En el contexto de la denominada Nueva Gestión Pública (NGP), Aguilar (2007) señala que las políticas públicas buscan hacer más asertivas las decisiones desde el Estado optimizando el proceso de gobernar. Se infiere directamente de esta vin- 
culación conceptual que las normativas, procedimientos y ejecución de éstas (implicadas en toda política pública) constituyen la forma concreta mediante la cual la gobernanza se actualiza plenamente.

La tercera noción que nos parece significativa en su vinculación con el concepto de gobernanza, es la de políticas territoriales. Se trata de una aportación de Müller (2002), quien plantea la existencia de políticas horizontales o territoriales en contraposición a la existencia de políticas verticales sectoriales. Las primeras (horizontales o territoriales) son llevadas a cabo por unidades territoriales dentro de una dimensión subnacional y en tal sentido de una gobernanza territorial local (en términos de Jolly, 2007, una gobernación del territorio). En este caso, se trataría de normativas y procedimientos que se ajustan de manera más real, pertinente y legítima a las necesidades y demandas de comunidades específicas dentro de un territorio mayor como el nacional. Desde nuestra metalectura del recorrido conceptual y los diversos giros y virajes que toma la noción de gobernanza, creemos que esta última vinculación es de gran relevancia ya que las políticas territoriales, entendidas en términos horizontales, permiten recoger y asumir en normativas concretas, variables culturales e idiosincrásicas desarrolladas desde las comunidades locales y en tal sentido, pueden dar cuenta de mejor manera de las múltiples demandas de esas comunidades.

En síntesis, estas tres vinculaciones al concepto de gobernanza, proyectadas a campos de sentido articulados desde la promoción de mejores políticas públicas, van dando cuenta de una teoría de la gobernanza que, como señala Mayntz (2000), considera a los contextos de producción de estas políticas, como una variable clave para su formulación. Efectivamente, Jessop (1998) advierte que la gobernanza debe hacerse cargo de las transformaciones que sufren las funciones del gobierno en un escenario de globalización/relocalización, cuestión que establece un desafío mayor para el buen gobierno en cualquiera de sus dimensiones (locales, regionales, nacionales, etc.). En este sentido, autores como Pierre y Peters (2000) y Meuleman (2009) definen la gobernanza como el conjunto de interacciones entre todo tipo de organismos públicos y privados además de la sociedad civil, que están orientadas a la resolución de los conflictos y problemas de comunidades concretas, comunidades de vida que se encuentran en coordenadas temporo-espaciales también muy definidas con cargas históricas e identitarias desde las cuales se tensionan, a través de conflictos y producen sus demandas para la solución de esos conflictos. Así, la variable contexto se abre paso como un elemento que condiciona de manera directa la articulación de un concepto vigoroso de gobernanza. En esta dirección, el desarrollo actual de una teoría de la gobernanza se sostiene desde una perspectiva epistémica compleja ya que en definitiva, el proceso de toma de decisiones para lograr determinados objetivos incluye una pluralidad muy amplia de variables, muchas de las cuales no pueden ser impuestas desde el gobierno central, sino que se encuentran en pleno desarrollo y evolución en los espacios locales, territoriales o micro- territoriales.

En el contexto latinoamericano, la producción teórica sobre el concepto de gobernanza ha sido más bien escasa. La instalación de este término en América Latina ha tenido que ver con entidades relacionadas a la cooperación internacional, tales como el Banco Mundial (BM), el Banco Interamericano de Desarrollo (BID), el Programa de las Naciones Unidas para el Desarrollo (PNUD) y sus proyectos de ayuda a los países subdesarrollados. Del mismo modo en que advertíamos (sección anterior), sobre una mayor y mejor gobernabili- 
dad en nuestra región y los peligros de la corrupción en ámbitos públicos y privados, con la gobernanza sucede lo mismo. Tanto el PNUD (UNDP1997) como el BM (1997), hacen notar la necesidad de transparencia y de procesos libres de corrupción para un mejor gobierno. Se hace necesario destacar también que las recomendaciones de este tipo de organismos internacionales son hechas dentro de la lógica de un modelo de Estado neoliberal y por tanto, los esfuerzos implicados en esta noción de gobernanza se orientan al fortalecimiento del libre mercado. La gobernanza aparece aquí ligada a reformas neoliberales que conduzcan a un pleno desarrollo, concepto adicional asociado a una reducción del Estado, liberación comercial, privatización de instituciones públicas y a fortalecer el derecho a la propiedad privada.

Si bien es cierto que la identidad ideológica de estos organismos es democrática-neoliberal y por tanto propende a un desarrollo basado en las fuerzas del mercado, es necesario destacar también que la puesta en práctica de una mejor gestión desde esta perspectiva, ha significado un proceso de descentralización en el cual se han generado como apuntan Daughters y Harper (2007), importantes políticas sociales focalizadas en los ámbitos de la salud, la educación y vivienda. Sin embargo, en dicho proceso descentralizador en el que se han promovido políticas sectoriales desde el paradigma neoliberal, no representa la constitución más genuina del formato conceptual en el que se expresa la gobernanza. Efectivamente, se ha creado un marco institucional para la democracia, pero dicho marco obedece a un traspaso acrítico de formatos para el buen gobierno. Esto significa que no se han dado los espacios para la construcción de una ciudadanía, como señalábamos antes, en consonancia y armonía con las reales aspiraciones de las sociedades. Las lógicas políticas institucionalizadas han terminado territorializándose, sin verdaderas consideraciones respecto al territorio en que dichas lógicas se ejercen. De este modo, la constitución de sociedades con mayores niveles de autonomía, organizadas por una gobernanza legítima y representativa, se tensiona y resuelve en conflictos que se hacen crónicos y que implican un largo trabajo social para poner en la agenda gubernamental mayor, las reales problemáticas que obstaculizan una mejor calidad de vida.

En síntesis, podemos señalar que el concepto de gobernanza y el campo semántico descrito y vinculado a este concepto, instalado en América Latina, no se ha traducido en una gestión orientada a más y mejor desarrollo de los pueblos latinoamericanos. El traspaso acrítico de un formato de gobernanza, concebido en Europa y Estados Unidos, a la realidad latinoamericana, constituye una debilidad fundamental. Como nos aporta Olsen (2006), esta instalación conceptual dogmática no ha considerado, entre otros aspectos, las relaciones de poder que ha establecido el Estado respecto a las sociedades involucradas, así como tampoco los procesos históricos a partir de los cuales se han concebido los instrumentos y políticas para gobernar. La falta o ausencia de una metalectura crítica respecto a las propuestas teóricas y operacionales sobre la acción concreta de hacer gobierno, ha permitido que la calidad institucional se mida en función al resguardo de los principios que rigen la economía de mercado.

Por las razones antes señaladas, es inevitable que América Latina como región y como conjunto de naciones, asuma el desafío de generar nuevos marcos conceptuales relativos a una gobernanza que se vincule a las necesidades reales de nuestras sociedades. Se trata de la expresión de arquitecturas nocionales emergentes cargadas de los contenidos culturales que nos definen y que en tal dirección deben apuntar a transformaciones estructurales profundas 
del Estado, así como a una comprensión distinta de la función del mercado y del desarrollo de la sociedad civil. Como hemos señalado antes, la gobernanza, el buen gobierno, se relacionan directamente con la optimización de la toma de decisiones en torno a la organización de la vida social y en tal sentido, la promoción de políticas públicas menos interesados en las instituciones que ostentan el poder (político, económico, militar, religioso, etc.) y por tanto, más preocupadas de los ciudadanos comunes y corrientes, debiesen orientarse a proyectos de desarrollo en los cuales las personas se sientan verdaderamente involucradas. Esto implica necesariamente que las perspectivas desde las que se desarrollan las normativas para una gobernanza más genuina deben incorporar en sus procesos analítico/metodológicos la mayor cantidad de variables que dan cuenta de la vida de una comunidad o sociedad. En otras palabras, la concepción de los formatos de gobernanza debe realizarse desde una lente compleja, para comprender e interpretar también un fenómeno complejo: el desarrollo de las culturas en cualquiera de sus dimensiones (local, subnacional, nacional, regional, etc.).

\section{Hacia una ( $r e$ comprensión compleja de los conceptos de gobernabilidad y gobernanza}

Como hemos expuesto hacia el final de la sección anterior, las nuevas teorías de la gobernanza y también las que tratan el concepto general de gobernabilidad, están forzadas a poner en acción una lectura crítica y aportativa que nutra dichos conceptos, no simplemente para dar más densidad semántica a los mismos, sino que para que se traduzcan efectivamente en mejores gobiernos. En este contexto, proponemos que tanto el concepto de gobernabilidad como el de gobernanza deben vincularse a la noción de complejidad, instalada como un aporte enriquecedor desde la instancia de la teorización sobre estos términos.

En tal dirección analítico/propositiva nos parecen interesantes las aportaciones críticas de Rottman (2002), a las cuales ya habíamos recurrido y que apuntan a una actitud de resistencia frente a una concepción de Estado excluyente y de una sociedad neoliberal que sólo conducen a nuevas formas de explotación y territorialización colonialista. En esta dirección diagnóstica, se trata de un desafío que se traduce en la necesidad de generar modelos alternativos de gobernabilidad y de gobernanza, profundizando en las múltiples lógicas que operan en la sociedad civil así como en las funciones del Estado que, como señala de Souza Santos (2004), representan articuladores legítimos para una redefinición de la democracia desde una posición contrahegemónica.

Ahora bien, desde esta mirada diagnóstica la gobernanza emerge como un concepto particularmente tensionado en su vinculación con realidades sociales específicas. Efectivamente, asociada a políticas concretas, a gobiernos locales y a su gestión en terreno, la gobernanza se enfrenta a una diversidad amplia de transformaciones sociales que requieren de su atención y de la resolución de las problemáticas contenidas en ellas.Variables tales como el crecimiento de la población y sus necesidades asociadas, la coexistencia de generaciones distintas, las diversas migraciones geográficas y culturales, las múltiples estructuras de familia y los diferentes procesos de segmentación social (que implican también segmentación territorial), constituyen algunas de las características del desarrollo social que los formatos de gobernanza deben incluir.

Desde nuestra perspectiva y frente a estos desafíos respecto a modelar formatos de gobernanza pertinentes a los contextos en que se implementarán, es necesario considerar a 
las sociedades y las transformaciones que estas sufren, como realidades complejas. Se trata de una consideración epistemológica que tiene como referente los trabajos de Morin (1977, 1986 entre otros), en los que este investigador da forma a un paradigma de la complejidad. De esta propuesta, nos interesan ciertos aspectos que a nuestro juicio, debiesen estar incorporados en toda teorización en el campo de las ciencias sociales, incluidas ciertamente aquellas de carácter sociopolítico, desde las que han emergido los conceptos de gobernabilidad y gobernanza.

En primer término, esta perspectiva epistémica nos permite comprender el hecho social y su desarrollo como un sistema complejo, es decir, en los términos de Morin (García, 2006), no reducible a un conjunto limitado de variables, situaciones o fenómenos que pueden ser aprehendidos en su totalidad y de una sola vez. Por el contrario, se trata de una comprensión que se despliega en un proceso de conocimiento en permanente construcción. En esta sentido, las sociedades y su desarrollo exigen ser concebidas desde morfologías particulares cargadas semánticamente de contenidos de mayor y menor significancia vinculados desde criterios que obedecen a lógicas diferentes y a variables de validación también diversas y distintas. Todo ello constituye una realidad compleja que se resiste a ser explicada desde un pensamiento simple (como el de la linealidad cartesiana) y que por tanto demanda un pensamiento complejo que vaya más allá de la simplificación, en la búsqueda o exploración de la mayor cantidad de elementos que son puestos en juego para articular las sociedades y las culturas que se generan y desarrollan desde y en la diversidad. Dicha diversidad de actores, materialidades y espacios, entre otras variables, a la luz de la complejidad no deben ser mutiladas con el fin ilusorio de construir ficciones operacionales orientadas a crear certidumbres que obviamente no dan cuenta del hecho social que se expresa dentro de un proceso histórico y contextual desde el que se nutre.

De este modo, el desafío actual de las teorías sociopolíticas para el planteamiento de conceptos como gobernabilidad y gobernanza, tiene que ver en primera instancia, con la comprensión de las sociedades y su desarrollo en términos complejos. Ya Durkheim (1937), intentó desarrollar planteamientos teóricos explicativos sobre la articulación de sociedades complejas. Lo mencionamos pues sus esfuerzos se pueden vincular con los planteamientos actuales sobre una sociedad compleja. Efectivamente, Durkheim visualiza el génesis de esta complejidad en las primeras congregaciones sociales expresadas en la horda. Esta forma social primitiva sería el antecedente evolutivo de todas las posteriores formas de organización social. La horda manifiesta una sociedad sin orden basada la mayor parte de las veces en acciones e intercambios no regulados ni menos normados. Desde ese diagnóstico, Durkheim intenta explicar la conformación de sociedades complejas como una sucesión de agregados ordenadores en una suerte de articulación recursiva a partir de una sociedad simple. Es así como el autor señala que en esta sucesión de agregados se produce la segmentación social que daría forma a una estructura más compleja de sociedad y que él denomina clan. (Durkheim, 1937). Desde la óptica de Durkheim, las sociedades se van volviendo más complejas en la medida en que desarrollan pliegues recursivos sobre sí mismas generando combinaciones de interacción más sofisticadas. En síntesis, la definición de la morfología social compleja en Durkheim, tiene que ver en primer término, con la sucesiva adición de agregados simples, pliegues de sentido que se van vinculando para, en una segunda instancia, dar forma a una arquitectura de mayor sofisticación que dé cuenta de la organización social. 
Esta descripción y explicación inicial dada por Durkheim respecto a la conformación de sociedades complejas, es enriquecida y actualizada desde la antropología contemporánea que tiene dentro de su plataforma epistemológica los principios de la complejidad moriniana. En este contexto, Mier Garza (1994) agrega que la complejidad social aumenta gracias a las nuevas formas de alianzas colectivas no normadas, el desarrollo de identidades individuales que se vinculan, a través de diversas modalidades a lo colectivo y múltiples dinámicas heterogéneas que van caracterizando en forma decisiva la estructura social y la consecuente densidad cultural que desde ella se produce.

La intelegibilización/comprensión de lo social como hecho complejo, (ya expuesta), ha significado simultáneamente la generación de un pensamiento teórico que se hace cargo de esa complejidad y que por tanto se abre, desde nuestra perspectiva (Brower, 2008), a las múltiples formas en que se vinculan sus variables o componentes y en consecuencia a la potencial estabilidad/inestabilidad de esas vinculaciones. En el contexto del proyecto teórico de Morin (1984, 1994), el pensamiento complejo debe reconstruir el proceso del conocimiento, incorporando las regularidades e irregularidades que se expresan en la sociedad y sus culturas debiendo describirse y explicarse como un fenómeno integral. La teorización sobre la complejidad social promueve una actividad analítica que, como hemos señalado en otro trabajo (Brower, 2008), acorte la distancia con lo que se conoce, advirtiendo en ese proceso de acercamiento, las articulaciones simbólicas mediante las cuales se expresa una cultura, consideración analítica que no debe ser entendida como definitiva.

Sin dudas, los principios que inspiran el pensamiento complejo debiesen ser considerados por las ciencias sociales en general y por la politología en particular. Como indica con lucidez Morin (2005), debemos ser capaces de enfrentar los desafíos del siglo XXI, respetando la diversidad humana, es decir, la multiculturalidad y su coexistencia en espacios o territorios comunes. El cultivo de la afectividad, de la fraternidad y capacidad de amar a través de una profunda comprensión del otro, constituyen un estado inicial básico para poner en marcha una modalidad de pensamiento (complejo) integral y no fragmentado. Estos principios/conceptos están ligados estrechamente con el arte de gobernar y por tanto con las nociones de gobernabilidad y gobernanza expuestas en las dos primeras secciones de este trabajo. Tanto las capacidades generales del buen gobierno como las políticas implementadas dentro de las naciones y aquellas que se proyectan a nivel regional y mundial debiesen considerar esta concepción de sociedad compleja ya que esta opción comprensiva implica necesariamente generar formatos de gobierno a partir de estrategias flexibles adaptables a concepciones muy diferentes de la vida.

De este modo, las advertencias críticas a las que hacíamos referencia al comienzo de esta sección, que tenían que ver con la necesidad de generar formatos de gobernanza desde espacios y tiempos locales, identificando e incluyendo las variables sobre las que se articulan y desarrollan las sociedades concretas, dan forma a una lente epistémica que permite entender las sociedades como sistemas complejos. Así, la resistencia expuesta por Rottman (2002), respecto a la concepción de Estados excluyentes que se aplican de forma acrítica en diferentes partes del mundo coincide con el planteamiento inicial de la comprensión compleja de la realidad social. De igual forma, nos parece relevante el aporte de Souza Santos (2004), al considerar la necesidad de entender las diversas lógicas que operan en el desarrollo/construcción de la sociedad civil, lógicas que además son expresadas en la dinámica social como 
los articuladores genuinos para la constitución de democracias contrahegemónicas capaces de establecer un diálogo válido con los sistemas dominantes generados básicamente desde Europa y Estados Unidos.

Ahora bien, como última consideración respecto al aporte de la lectura compleja sobre los formatos conceptuales de gobernabilidad y gobernanza, es necesario señalar en la dirección de entender e incluir lógicas diferentes en la articulación y desarrollo de las sociedades, que tanto la conceptualización como los protocolos de implementación de políticas públicas que aseguren gobernabilidad y una gobernanza genuina respecto a las necesidades y demandas de la sociedad, sean asumidas desde una mirada interdisciplinaria, cuestión o exigencia también insoslayable para la comprensión de sistemas complejos. Como hemos señalado en otros trabajos (Brower, 2008,2010,2011), la interdisciplinariedad permite que diversas teorías y metodologías dialoguen sobre tópicos concretos con el fin de comprender de mejor manera la trama inabarcable de la cultura. Por su parte, Sinaceur (1983) considera la interdisciplinariedad como una instancia en la que convergen puntos de vista diferentes, haciendo que los expertos den una visión limitada sobre un tema que, al interactuar con otros puntos de vista, articulan o dan forma a una síntesis explicativa enriquecida sobre dicho tema.

De esta forma, entender y vincular las sociedades y su desarrollo con la concepción interdisciplinaria del conocimiento, puede traducirse, como un proceso gnoseológico desplegado desde la lógica de la complejidad. Asumir esta clave conceptual de lectura, significa a su vez desde nuestra óptica epistemológica, mejorar y optimizar el ejercicio del buen gobierno ya que permite enriquecer notablemente la conceptualización respecto a la gobernabilidad y la gobernanza.

\section{Conclusiones: fin de un viaje conceptual inacabado}

Una vez concluido este recorrido conceptual sobre los términos gobernabilidad y gobernanza, recorrido sin pretensiones de exhaustividad y que por tanto sólo se ha detenido en aquellos campos semánticos que nos han parecido interesantes para darles mayor espesor semántico, podemos fijar ciertas vinculaciones de sentido que pueden cooperar en su lectura y comprensión.

En primer lugar, los conceptos gobernabilidad y gobernanza se han utilizado en ocasiones en forma indistinta, para referirse al ejercicio del buen gobierno y las competencias que ese ejercicio implica, sin embargo una revisión cuidadosa de la producción teórica al respecto va diseñando límites o fronteras entre ambos que se hace necesario destacar. Es así como la gobernabilidad aparece ligada a ciertas capacidades más bien generales para gobernar, capacidades que a su vez, se entienden o se condicionan dentro de un marco político democrático y en consonancia directa con el modelo económico de mercado neoliberal. Este cluster conceptual (conjunto de vinculaciones y asociaciones entre términos), es problematizado desde las ciencias políticas o la politología general en torno a una preocupación central: el debilitamiento de los sistemas democráticos en el mundo (en particular el tercer mundo) y la necesidad urgente de fortalecerlos. Es necesario advertir en este cruce problemático conceptual, que el interés teórico insiste en una mayor territorialización/validación de la democracia, pero fuertemente ligado a las economías de libre mercado. No es extraño entonces que organismos internacionales como el Banco Mundial sean los que 
emitan mayor cantidad de reportes e informes sobre la situación de las economías europeas, africanas y latinoamericanas. En efecto, desde esta perspectiva, dichos informes ligan el funcionamiento del mercado con el estado de salud de las democracias ya sean emergentes o más consolidadas. Este embrague o conexión/combinación de conceptos nutren el término gobernabilidad. Por último, siempre aparece en esta concepción democrática neoliberal el tema/tópico de la corrupción, pues este fenómeno altera el flujo legal del mercado, genera pérdidas, elusión de impuestos y blanqueo de dineros que provienen de mercados clandestinos como el narcotráfico entre otros.

Por otro lado, el concepto de gobernanza ha tenido en las últimas décadas un desarrollo y enriquecimiento semántico muy interesante. Este término no se refiere a la capacidad o capacidades generales para gobernar sino que se vincula directamente a las políticas públicas que se implementan para la organización de las sociedades y por otro lado para satisfacer las demandas de la sociedad civil, cada vez más amplias y con exigencias de respuesta más rápidas. Planteada así, la gobernanza se comprende dentro de marcos constitucionales definidos y estables y se vincula a un conjunto de leyes que permitan garantizar una buena distribución del poder y a normativas reglamentarias a través de las cuales estas leyes se ejecuten.

En este contexto, la problematización teórica respecto a la noción de gobernanza se ha instalado en forma crítica, respecto a la tarea de generar formatos para la gobernanza que se adecuen a territorios específicos, recogiendo las múltiples variables identitarias y de desarrollo con las que las comunidades cuentan para proyectarse al futuro. En tal dirección, las políticas públicas aparecen como la expresión clave que debiese contener desde esta mirada analítica/crítica, las lógicas locales desde donde se entienden conceptos como progreso o desarrollo. Este tópico representa un punto de inflexión dentro del debate teórico sobre la gobernanza. De hecho, hemos podido comprobar cómo en América Latina los investigadores de esta área cuestionan los formatos de gobernanza trasladados acríticamente desde Europa a nuestras tierras. Se visualiza en nuestro recorrido conceptual esta problematización que implica un cuestionamiento al propio concepto de democracia y claramente a la economía de libre mercado.

De este modo podemos concluir que las teorías políticas se enfrentan, cada vez con mayor intensidad, a la exigencia/demanda de la sociedad civil, en el sentido de articular o construir políticas públicas que la represente y que por tanto dé cuentan de los contenidos que importan a dicha sociedad. Allí se encuentra el punto central de los nuevos formatos de gobernanza y por tanto de su conceptualización más profunda y genuina.

Frente a este desafío finalizamos nuestro trabajo con una sección que conecta el recorrido conceptual sobre gobernabilidad y gobernanza con una avenida de sentido que no se encuentra en una dimensión teórica, su no que más bien aporta una lente o perspectiva epistemológica para el enriquecimiento conceptual de términos como estos, particularmente al referido a la gobernanza. Se trata de la perspectiva compleja o mirada desde la complejidad, cuyos principios hacen claridad respecto a la necesidad de comprender la mayor cantidad de variables en la articulación y desarrollo de las sociedades entendidas por esto, como un hecho complejo. La complejidad en este contexto no debe ser entendida como un fenómeno difícil de explicar o inaccesible. Por el contrario, esta propuesta epistémica amplía los horizontes comprensivos de la realidad social, potenciando en el caso específico del acto de gobernar, su conceptualización y su traducción en la dimensión práctica de hacer 
un mejor gobierno. Esta vinculación/conexión con los principios de la complejidad queda aquí como una propuesta inicial y por tanto inacabada que es necesario profundizar a través de un diálogo interdisciplinario que finalmente sea capaz de producir mejores formas de organización de la vida en sociedad.

\section{Referencias bibliográficas}

Aguilar, L.F. 2007. "El aporte de la política pública y de la nueva gestión pública a la gobernanza". En Revista del CLAD Reforma y Democracía. №39, Caracas.

Banco Mundial. 1989. "El África Subsahariana: De la Crisis al Desarrollo sustentable. Una perspectiva de largo plazo". D.C. BM/Oxford University Press.

Banco Mundial. 1997. Informe sobre desarrollo humano 1997. El Estado en un mundo en transformación. Washington, D.C. BM/Oxford University Press.

Brower, J.2008. "Implicancias epistemológicas del pensamiento complejo para la articulación de la una semiótica de la cultura". En Revista Estudios de Filosofía N³7. Universidad de Antioquia. Medellín. Colombia.

Brower, J. 2010. "El valor semiótico y Dialógico de las nuevas epistemologías”. En Revista Observaciones Filosóficas. N⒑ Universidad Católica de Valparaíso.

Brower, J. 2011. "Convergencias epistemológicas para la producción de conocimiento en el ámbito de las Ciencias Sociales: hacia la delimitación del valor semiótico y dialógico de las nuevas epistemologías". En Revista Re-Presentaciones. Periodismo, Comunicación y Sociedad. Año IV. N8. Universidad de Santiago de Chile.

Crozier, M.J., Huntington, S.P., Watanuki, J., 1975. The Crisis of Democracy: Report on the Gobernability of Democracies to the Trilateral Commission. New York University Press.

Dahl, R.A. 1998. Democracy. Yale University Press.

Durkheim, É. 1937. Les regles de la méthode sociologique. París, PUF.

Daughters, R. y Harper L. 2007. "Fiscal and Political Decentralization Reforms". En Eduardo Lora (ed.), The State of State reform in Latin America. Washington, D.C., BID/ BM/Standford University Press.

De Souza Santos, B. 2004. Democratizar la Democracia: Los caminos de la democracia participativa. México,FCE.

García, R. 2006. Sistemas complejos. Conceptos, métodos y fundamentación epistemológica de la investigación interdisciplinaria. Barcelona, Gedisa.

Gaudin, J.P. 2002. Pourquoi la gouvernance?. Paris, Presses de Sciences Po.

Huntington, S.1972. El orden político en las sociedades en cambio. Buenos Aires, Paidos.

Huntington, S. 1991. The Third Wave: Democratization in the Late Twentieth Century. University of Oklahoma Press.

Jessop, B. 1998. "The Rise of Governance and the Risks of Failure: The Case of Economic 5. Development”. International Sociaql Science Journal. Vol. 50, $\mathrm{n}^{\circ} 155$.

Jiménez, W.,Ramírez, C., Roncancio, P. 2007. Gobernabilidad y gobernanza en la transformación de la acción pública: propuesta teórica y estudio de caso. Bogotá, ESAP Publicaciones.

Jolly, J.-F. 2007. “Gobernabilidad territorial y descentralización en Colombia: ¿regir el territorio o gobernar los territorios? A propósito de la política pública de descentralización 
en Colombia entre 1982 y 2002". Conferencia expuesta en el Foro Internacional "Globalización, Gobernabilidad y Territorio". Escuelas Superior de Administración Pública. Bogotá, 29 y 30 de viembre.

Kaufmann, D. 2002-2003. Rethinking Governance. En Reporte Global de Competitividad. Mayntz, R. 2000. "Nuevos desafíos de la teoría de governance". En Instituciones y Desarrollo. $\mathrm{N}^{\circ} 7$. Barcelona.

Meuleman, L. 2009. "The Cultural Dimension of Metagovernance: Why Governance Doctrines May Fall”. Public Organization Review. DOI: 10.1007s11115-009-0088-5.

Mier Garza, R. 1994. Vicisitudes de la inestabilidad: apuntes para una reflexión sobre la noción de complejidad en antropología. Boletín de Antropología Americana. Instituto Panamericano de Geografía e Historia. N²9.

Morin, E. 1977. Tomo 1. La nature de la nature. En La Méthode. París: Editions du Seuil.

Morin, E. 1984. Ciencia con conciencia. Barcelona, Anthropos.

Morin, E. 1994. Introducción al pensamiento complejo. Barcelona, Gedisa.

Morin, E. 1986. Tomo 3. La connaissance de la connaissance. En La Méthode. París: Editions du Seuil.

Morin, E. 2005.Esperando nuestra mariposa. Iniciativa Socialista. $\mathrm{N}^{\circ} 77$. Disponible en: http://www.inisoc.org/. Accesio el 11 de abril de 2007.

Muller, P. 2002. Las políticas públicas. Bogotá. Universidad Externado de Colombia.

Olsen, J.P.2006.'Maybe It Is Time to Rediscover Bureaucracy”. Journal of Public Adminsitration Research and Theory. Vol. 16. $\mathrm{N}^{\circ} 1$.

Pierre, J. y Peters, G. 2000. Governance, Politics and the State. Basingstoke, Macmillan.

Prats i Catala, J. 2001. "Gobernabilidad democrática para el desarrollo humano: marco conceptual y analítico”. En Instituciones y Desarrollo, №10, Barcelona.

Prats i Catalá, J. 2003. "El concepto y el análisis de la gobernabilidad". En Instituciones y Desarrollo. $\mathrm{N}^{\circ}$ 14-15, Barcelona.

Rottman, M. 2002. La gobernabilidad: ¿ un problema teórico-práctico?. En Salinas, D. y Jiménez, E. (coordinadores), Gobernabilidad y Globalización. Procesos recientes en América Latina. México, Gernica.

Sinaceur, M.A. 1983. ¿Qué es interdisciplinariedad?. En Apostel, L. et al. Interdisciplinariedad y ciencias humanas. Madrid, Tecnos/Unesco.

UNDP, 1997. Governance for Sustainable Human Development. Nueva York, UNDP.

Zurbriggen, C. 2003. "Las redes de políticas públicas: una revisión teórica”. Barcelona, Instituto internacional de Gobernabilidad de Cataluña (Colección Documentos). 